\title{
2013 Veblen Prize
}

IAN AGOL and DANIEL WISE received the 2013 Oswald Veblen Prize in Geometry at the 119th Annual Meeting of the AMS in San Diego, California, in January 2013.

\section{Citation for lan Agol}

The 2013 Oswald Veblen Prize in Geometry is awarded to Ian Agol for his many fundamen-

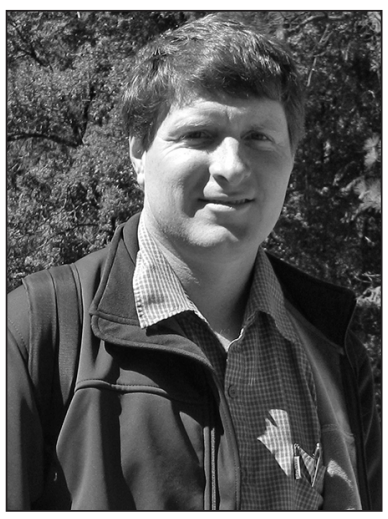

tal contributions to hyperbolic geometry, 3-manifold topology, and geometric group theory. In particular, Agol is cited for the following papers:

(with P. Storm and W. P. Thurston) "Lower bounds on volumes of hyperbolic Haken 3-manifolds" with an appendix by Nathan Dunfield, Journal of the American Mathematical Society 20 (2007), no. 4, 1053-1077.

"Criteria for virtual fibering", Journal of Topology 1 (2008), no. 2, 269-284.

(with D. Groves and J. F. Manning) "Residual finiteness, QCERF and fillings of hyperbolic groups", Geometry and Topology 13 (2009), no. 2, 1043-1073.

In 2004 Agol (and independently Calegari and Gabai) proved the Marden tameness conjecture, which led to many important results in hyperbolic geometry, among them the Ahlfors measure conjecture.

Agol subsequently proved a number of important results on volumes of hyperbolic 3-manifolds, including an important inequality, found with Nathan Dunfield in the first cited paper, relating the volume of a closed hyperbolic 3-manifold with that of a drilled manifold and the tube radius of the drilled geodesic. This played an important role in the proof (by Gabai, Meyerhoff, and Milley) that the Weeks manifold is the unique lowest volume closed orientable hyperbolic 3-manifold.

In the second paper Agol found a key group theoretic criterion for a closed irreducible 3-manifold to have a finite sheeted covering space that fibers over the circle. With Daniel Groves and Jason Manning, he showed in the third paper that if all hyperbolic groups are residually finite, then any

DOI: http://dx.doi.org/10.1090/noti973 quasiconvex subgroup of a hyperbolic group is separable.

In April 2012 Agol posted an article to the arXiv announcing a proof of a conjecture of Daniel Wise implying both Waldhausen's virtual Haken conjecture and Thurston's virtual fibering conjecture. The proof involves a generalization of the methods of these papers, clever new ideas, and it makes crucial use of results of Wise and joint work of Haglund and Wise. The resolution of these conjectures is a milestone achievement in the theory of 3-dimensional manifolds.

\section{Biographical Sketch}

Ian Agol was born in Hollywood, California, in 1970 and received his Ph.D. at the University of California San Diego in 1998 under the supervision of Michael Freedman. He held postdoctoral positions at UC Davis and the University of Melbourne before teaching at the University of Illinois at Chicago. He moved to UC Berkeley in 2007. He received a Guggenheim fellowship in 2005 and spoke at the International Congress of Mathematicians (ICM) in Madrid (2006).

He shared the 2009 Clay Research Award with Danny Calegari and David Gabai for the solution of the Marden tameness conjecture. He received the 2012 Senior Berwick Prize for his cited paper, "Criteria for virtual fibering".

\section{Response}

It is an honor to share the 2013 Oswald Veblen Prize in Geometry with Dani Wise.

I owe a debt of gratitude to my family for their support, especially my mother, who made sure that I had a top-notch education and encouraged me to pursue a career in a field I am passionate about, and my wife, Michelle, for her support. I also share this award in spirit with my collaborators, especially Nathan Dunfield, Peter Storm, Daniel Groves, Jason Manning, and the late Bill Thurston, with whom I collaborated on the papers mentioned in the citation. My research on the virtual Haken conjecture was influenced heavily by the work of Dani Wise and his collaborators Nicolas Bergeron, Tim Hsu, Michah Sageev, and especially Frédéric Haglund. Without Wise's insights, this approach to the conjecture would have been impossible, and his vision completely changed my perspective on the problem. Another key contribution was made 
by Jeremy Kahn and Vlad Markovic on the surface subgroup problem. The subject of hyperbolic groups and CAT(0) cube complexes, introduced by Misha Gromov, has reached a mature state, which I think is now made evident by the first Veblen Prize given to a geometric group theorist after Gromov himself.

In the 1980s, Bill Thurston laid out his vision for three-dimensional topology and Kleinian groups. Over the past thirty years, his vision has been validated, with such milestones achieved as the geometrization theorem, the ending lamination theorem, the tameness theorem, the density theorem, and now the virtual fibering theorem. The virtual Haken theorem (originating as a question of Waldhausen) is a purely topological statement; however, its resolution is in some sense mostly geometrical and depends on many developments from outside the field, including PDEs, Riemannian and Alexandrov geometry, dynamics, representation theory, and geometric group theory.

I hope that 3-manifolds and Kleinian groups will give back equally to these and other subjects in the future. Moreover, I think the most important project for the future is to make connections between this highly developed area of geometric 3-manifold topology and other approaches, such as various flavors of Floer homology, topological quantum field theory, knot theory and 4-manifolds, and other types of geometric structures on 3-manifolds.

\section{Citation for Daniel Wise}

The 2013 Veblen Prize in Geometry is awarded to Daniel Wise for his deep work establishing subgroup separability (LERF) for a wide class of groups and for introducing and developing with Frédéric Haglund the theory of special cube complexes, which are of fundamental importance for the topology of 3-dimensional manifolds. In particular Wise is cited for the following papers:

"Subgroup separability of graphs of free groups with cyclic edge groups", Quarterly Journal of Mathematics 51 (2000), no. 1, 107-129. A subgroup is separable if it is the intersection of all the finite index subgroups that contain it. This is an important condition in 3-manifold topology since up to homotopy an immersed surface in a closed irreducible 3-manifold lifts to an embedded surface in some finite sheeted cover if the immersion induces an isomorphism of the fundamental group of the surface with a separable subgroup of the fundamental group of the 3-manifold.

"Residual finiteness of negatively curved polygons of finite groups", Inventiones Mathematicae 149 (2002), no. 3, 579-617. This paper is representative of a long arc of work in which Wise describes powerful and ingenious criteria for showing that a group is residually finite or, more generally, that all of its quasiconvex subgroups are separable. (with F. Haglund) "Special cube complexes", Geometric and Functional Analysis 17 (2008), no. 5, 1551-1620.

(with F. Haglund) "A combination theorem for special cube complexes", Annals of Mathematics 176 (2012), no. $3,1427-1482$. In the first of these papers, Wise and Haglund introduced the concept of a "special cube complex". The fundamental groups of such complexes have various

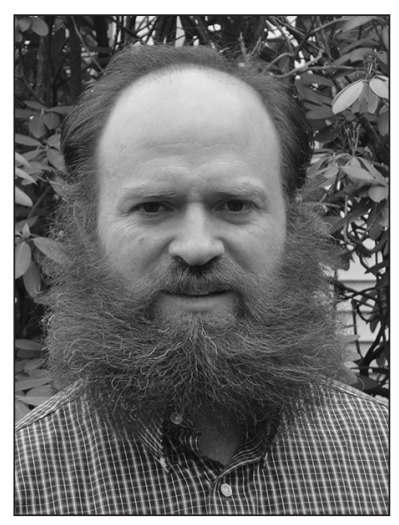

Daniel Wise remarkable properties. For example, they embed into right-angled Artin groups and their quasiconvex subgroups are separable. Generalizing the methods of the two papers of Wise cited above, Wise and Haglund show that a group obtained from two virtually special hyperbolic groups that are amalgamated along quasiconvex malnormal subgroups is virtually special. In subsequent work Wise used this to prove that the fundamental groups of Haken hyperbolic 3-manifolds are virtually special.

Beyond what is cited in these papers, Wise made the far-reaching conjecture that a Gromov hyperbolic group that acts cocompactly and properly on a CAT(0) cube complex is the fundamental group of virtually special cube complexes. Ian Agol proved this conjecture making essential use of the technology of Wise and his collaborators. Combined with the work of Jeremy Kahn and Vladimir Markovic, and with the work of Michah Sageev, this gives a positive solution to the long-standing Waldhausen virtual Haken conjecture.

\section{Biographical Sketch}

Daniel Wise was born in 1971 and grew up in the New York City area. He received a B.A. in 1991 from Yeshiva College and a Ph.D. in 1996 from Princeton University. After stints at UC Berkeley, Cornell University, and Brandeis University, he joined McGill University, where he has been teaching since 2001. Dani lives in Montreal with his wife, Yael, and their four children. Their house is full of music, art, laughter, and sleep deprivation.

\section{Response}

I am grateful to be the recipient, together with Ian Agol, of the Oswald Veblen Prize of the AMS. I share my part of this recognition with my coauthors Nicolas Bergeron, Chris Hruska, Tim Hsu, Michah Sageev, and especially Frédéric Haglund. This is a welcome opportunity to thank Jonathan Sondow and Sylvain Cappell, who launched me into mathematics, Martin Bridson, who initiated my interest in geometric group theory, my colleagues 
and students at McGill, my friends and family, and my wife, Yael.

I was attracted to residual finiteness at the very beginning of my career-and latched on to the idea of a "clean complex" in 1993. From that point onward I progressively nurtured a belief that a variant of this was a key toward understanding basic properties of fundamental groups of 3-manifolds. I made progress in 1997 toward understanding that many prime alternating link groups are virtually fundamental groups of clean complexes. In 2000, I realized that most small-cancellation groups had codimension-one subgroups, made the connection to Michah Sageev's work on dual CAT(0) cube complexes, and then began a crusade to "cubulate" all groups in sight. To my great fortune, Frédéric Haglund visited in 2002, and we developed the notion of a "special cube complex". These "generalized graphs" improved upon clean complexes in a manner that I had only dreamed about beforehand. My previous work was readapted in this more elegant context with no 2-dimensional limitation, and this began to catch the attention of the geometric group theory community. There was now something concrete to aim for-and at the 2004 Spring Topology and Dynamics conference, I laid out a plan for understanding groups: first cubulate and then find a special cover. I explained that my conjecture, that each hyperbolic 3-manifold $M$ is virtually special, is equivalent to the pair of well-known conjectures that $\pi_{1} M$ has separable quasiconvex subgroups and that $\pi_{1} M$ has sufficiently many quasiconvex surface subgroups (now a recent accomplishment of Kahn and Markovic). Though some topologists were reluctant to believe in the cubical route, by 2005 I had mapped out a strategy for proving a significant case: the virtual specialness of "hyperbolic groups with a quasiconvex hierarchy". The various parts of this plan were accomplished with the great help of my collaborators, and during my 2008-2009 sabbatical at the Hebrew University, I was able to write up details of this work. I remain deeply grateful to Zlil Sela for his feedback and encouragement during that time. It brings tremendous satisfaction to have seen Ian Agol bring this program to completion among his many great achievements.

\section{About the Prize}

The Veblen Prize is awarded every three years for a notable research memoir in geometry or topology that has appeared during the previous five years in a recognized North American journal (until 2001 the prize was usually awarded every five years). Established in 1964, the prize honors the memory of Oswald Veblen (1880-1960), who served as president of the AMS during 1923-1924. It carries a cash award of US\$5,000.

The Veblen Prize is awarded by the AMS Council acting on the recommendation of a selection committee. For the 2010 prize, the members of the selection committee were Tobias H. Colding, William G. Dwyer, and Michael J. Hopkins.

Previous recipients of the Veblen Prize are: Christos D. Papakyriakopoulos (1964), Raoul H. Bott (1964), Stephen Smale (1966), Morton Brown and Barry Mazur (1966), Robion C. Kirby (1971), Dennis P. Sullivan (1971), William P. Thurston (1976), James Simons (1976), Mikhael Gromov (1981), Shing-Tung Yau (1981), Michael H. Freedman (1986), Andrew Casson (1991), Clifford H. Taubes (1991), Richard Hamilton (1996), Gang Tian (1996), Jeff Cheeger (2001), Yakov Eliashberg (2001), Michael J. Hopkins (2001), David Gabai (2004), Peter Kronheimer, Tomasz Mrowka, Peter Ozsváth, and Zoltán Szabó (2007), Tobias H. Colding and William P. Minicozzi (2010), and Paul Seidel (2010). 